\title{
ASYMPTOTIC VALUES AND BAIRE CATEGORY
}

\author{
CHAIM MIDA
}

Abstract. Let $f$ be meromorphic in the unit disc, and let $\alpha$ be a complex number. Given $\varepsilon>0$, let $T_{\varepsilon}(\alpha)$ denote the set of points $e^{i \theta}$ for which the cluster set $C_{\mathscr{L}}\left(f, e^{i \theta}\right)$ lies in the $\varepsilon$-neighbourhood of $\alpha$ for some arc $\mathscr{L} \rightarrow e^{i \theta}$. Then a sufficient condition that the set of points on the unit circle at which $f$ possesses point-asymptotic value $\alpha$ be of first category is that $T_{\varepsilon}(\alpha)$ contains no arc for some $\varepsilon>0$.

1. Introduction. Let $f$ be meromorphic in the unit disc $D:|z|<1$. Given a complex number $\alpha$, the set of points on the unit circumference $C$ at which $f$ possesses $\alpha$ as a point-asymptotic value is denoted by $\Gamma(\alpha)$. In this paper we shall give a sufficient condition in order that $\Gamma(\alpha)$ be of first category on $C$.

For a point $e^{i \theta}$ of $C$, we shall write $\mathscr{L} \rightarrow e^{i \theta}$ to mean that $\mathscr{L}$ is a simple arc lying in $D$ except for one endpoint at $e^{i \theta}$. If $\mathscr{L} \rightarrow e^{i \theta}$, then we let $C_{\mathscr{L}}\left(f, e^{i \theta}\right)$ denote the cluster set of $f$ at $e^{i \theta}$ along $\mathscr{L}$. Given a point $\alpha$ of the extended plane $W$ and any $\varepsilon>0$, we let $N_{\varepsilon}(\alpha)=\{z|| z-\alpha \mid<\varepsilon\}$ for finite $\alpha$, while $N_{\varepsilon}(\infty)=\{z|| z \mid>\varepsilon\}$. Then for any point $\alpha$ of $W$ and any $\varepsilon>0$ we define the set $T_{\varepsilon}(\alpha)$ as follows:

$$
T_{\varepsilon}(\alpha)=\left\{e^{i \theta} \mid \text { there exists } \mathscr{L} \rightarrow e^{i \theta} \text { with } C_{\mathscr{L}}\left(f, e^{i \theta}\right) \subset N_{\varepsilon}(\alpha)\right\} .
$$

2. The Baire category of $\Gamma(\alpha)$. In general, the set $\Gamma(\alpha)$ need not be of first category on $C$. Indeed, we shall exhibit a function, bounded and analytic in $D$, with constant angular limits at a subset of $C$ of second category.

Consider a set $J$ of measure zero and second category on $C$. Then there exists [3, p. 369] a nondecreasing absolutely continuous function $\mu(\theta)$ on $C$ such that $\mu^{\prime}(\theta)=\infty$ at every point of $J$, and such that the total variation of $\mu(\theta)$ is less than 1 . Then the Stieltjes integral

$$
P(z)=\frac{1}{2 \pi} \int_{0}^{2 \pi} \frac{1-r^{2}}{1+r^{2}-2 r \cos (\psi-\theta)} d \mu(\theta), \quad z=r e^{i \varphi},
$$

represents a positive harmonic function in $D$. By Fatou's theorem, $P$

Received by the editors February 26, 1973.

AMS (MOS) subject classifications (1970). Primary 30A72.

(c) American Mathematical Society 1973 
possesses angular limit $\infty$ at every point of $J$. Thus, if we let $Q$ denote a harmonic conjugate of $P$ in $D$, and set $w=P+i Q$, then the function $f$ defined by $f=e^{-w}$ is readily seen to be analytic and bounded in $D$ with angular limit zero at every point of $J$. An example of this type was first constructed by Lusin and Privalov.

We now proceed to the principal result of this paper.

THEOREM. Let $f$ be meromorphic in $D$ and let $\alpha$ be any complex number. If there exists $\varepsilon^{*}>0$ such that $T_{\varepsilon^{*}}(\alpha)$ does not contain an arc of $C$, then $\Gamma(\alpha)$ is of the first category on $C$.

Proof. Without loss of generality we may assume that $\alpha=\infty$, for otherwise we may consider the function $1 /(f-\alpha)$. Choose $\sigma>\varepsilon^{*}$ and let $H_{\sigma}=\{z|| f(z) \mid>\sigma\}$. If $\Gamma(\infty)=\varnothing$ there is nothing to prove. So, we assume that $\Gamma(\infty) \neq \varnothing$ and hence $H_{\sigma} \neq \varnothing$. Let $G_{\sigma}$ be a nonempty component of $H_{\sigma}$ and suppose further that both the sets $M_{\sigma}=\operatorname{Fr} G_{\sigma} \cap C$ and $\Gamma_{\sigma}=\Gamma(\infty) \cap M_{\sigma}$ are nonempty.

We first establish that the set $G_{\sigma} \cup\{p\}$ is locally connected for any point $p$ of $\Gamma_{\sigma}$. Since $G_{\sigma}$ is a locally connected domain it follows that if $G_{\sigma} \cup\{p\}$ is not locally connected at $p$ then there exists a sequence of continua $\left\{K_{n}\right\}$ of $\operatorname{Fr} G_{\sigma} \cap D$ tending to a limiting nondegenerate continuum $K$ containing $p$ in its interior. Furthermore, as $\operatorname{Fr} G_{\sigma}$ is clearly locally connected at any of its points which lie in $D, K$ must be a closed arc of $C$. Since $p$ lies in $\Gamma_{\sigma}$, there exists a point $q$ interior to $K$ and a simple arc $\mathscr{L}_{a} \rightarrow q$ with $f \rightarrow \infty$ along $\mathscr{L}_{a}$. However, since $|f(z)|=\sigma$ at all points of any $K_{n}$, and $\mathscr{L}_{a}$ must necessarily cross infinitely many of the continua $\left\{K_{n}\right\}$ arbitrarily close to $q, C_{\mathscr{L}_{Q}}(f, q)$ contains points of modulus $\sigma$, which is not possible. Thus, $G_{\sigma} \cup\{p\}$ is locally connected for any point $p$ of $\Gamma_{\sigma}$, and hence [4, p. 111] each point of $\Gamma_{\sigma}$ is then arcwise accessible from $G_{\sigma}$.

It follows from the above that for each point $p$ of $\Gamma_{\sigma}$ we can construct an $\operatorname{arc} \mathscr{L}_{p} \rightarrow p$ with $C_{\mathscr{L}_{p}}(f, p) \subset N_{\varepsilon^{*}}(\infty)$ (as $\left.\sigma>\varepsilon^{*}\right)$. Thus, by our hypothesis concerning $T_{\varepsilon^{*}}(\infty)$, it follows that $\Gamma_{\sigma}$ cannot contain an arc of $C$, and so $\Gamma_{\sigma}$ is nowhere dense on $C$. Now if $p$ is any point of $\Gamma(\infty)$, then there exists $\mathscr{L}_{p} \rightarrow p$ with $f \rightarrow \infty$ along $\mathscr{L}_{p}$. Hence, some terminal part of $\mathscr{L}_{p}$ must lie completely in one component $G_{\sigma}^{\prime}$ of $H_{\sigma}$ with $p$ in $\Gamma_{\sigma}^{\prime}=\Gamma(\infty) \cap$ $M_{\sigma}^{\prime}\left(M_{\sigma}^{\prime}=\operatorname{Fr} G_{\sigma}^{\prime} \cap C\right)$. The theorem now follows from the observation that $H_{\sigma}$ has at most countably many components.

We remark that, although $\Gamma(\alpha) \subseteq T_{\varepsilon}(\alpha)$ for all $\varepsilon>0$, the above theorem involves no restriction on the measure of $T_{\varepsilon^{*}}(\alpha)$ and hence $\Gamma(\alpha)$ may in general be of positive linear measure.

In particular, a sufficient condition in order that $\Gamma(\alpha)$ be of first category on $C$ is that $m\left(T_{\varepsilon^{*}}(\alpha)\right)=0$ for some $\varepsilon^{*}>0$. By combining this observation 
with the ambiguous-point theorem (see for example [1, p. 83]) we may obtain results for some known classes of functions. For example, if $f$ is a function of class $U^{*}$; that is,

$$
\lim _{r \rightarrow 1}\left|f\left(r e^{i \theta}\right)\right|=1
$$

almost everywhere on $C$, then $m\left(T_{\varepsilon^{*}}(\infty)\right)=0$ for any $\varepsilon^{*}>1$ and hence $\Gamma(\infty)$ is of first category on $C$. More generally, we state the following

COROLlaRY. Let $f$ be meromorphic in $D$ and suppose that for some finite point $\alpha$ of $W$ there exists $k>0$ such that $m\left(T_{k}(\alpha)\right)=2 \pi$. Then $\Gamma(\infty)$ is of first category on $C$.

As a final application we consider a nonconstant inner function in $D$. For a point $\alpha,|\alpha|<1$, letting $0<\varepsilon^{*}<1-|\alpha|$ we see that $m\left(T_{\varepsilon^{*}}(\alpha)\right)=0$. Since for any point $\alpha,|\alpha|=1$, the set of points on $C$ at which $f$ possesses radial limit $\alpha$ is the same as that for which the inner function $\exp \{(f+\alpha) /(f-\alpha)\}$ possesses radial limit zero, we obtain the following recent result [2].

COROLlaRY. Let $f$ be a nonconstant inner function in $D$. Then the set of points on $C$ at which a given complex number $\alpha$ is a radial limit of $f$ is of first category.

\section{REFERENCES}

1. E. F. Collingwood and A. J. Lohwater, The theory of cluster sets, Cambridge Tracts on Math. and Math. Phys., no. 56, Cambridge Univ. Press, Cambridge, 1966. MR 38 \#325.

2. A. J. Lohwater, Some function-theoretic results involving Baire category, Jyväskylä Conference on Analysis (Jyväskylä, Finland, 1970), Springer-Verlag, 1972.

3. E. C. Titchmarsh, The theory of functions, 2nd ed., Oxford Univ. Press, Oxford, 1939.

4. G. T. Whyburn, Analytic topology, Amer. Math. Soc. Colloq. Publ., vol. 28, Amer. Math. Soc., Providence, R.I., 1942. MR 4, 86.

Department of Mathematics, University of Toronto, Toronto, Ontario, CANADA 